\title{
Iterative Detection and Decoding with PIC Algorithm for MIMO-OFDM Systems
}

\author{
Zhongpeng WANG \\ School of Information Technology and Electronic Engineering \\ Zhejiang University of Science and Technology, Hangzhou, China \\ Email:wzp1966@sohu.com \\ Received February 4, 2009; revised March 25, 2009; accepted April 30, 2009
}

\begin{abstract}
In this paper, we propose a new iterative detection and decoding scheme based on parallel interference cancel (PIC) for coded MIMO-OFDM systems. The performance of proposed receiver is improved through the joint PIC MIMO detection and iterative detection and decoding. Its performance is evaluated based on computer simulation. The simulation results indicate that the performance of the proposed receiver is greatly improved compared to coded MIMO-OFDM systems based on VBLAST detection scheme.
\end{abstract}

Keywords: Iterative Detection and Decoding, MIMO-OFDM, PIC Signal Detection, VBLAST

\section{Introduction}

In multipath environments, multiple input multiple output wireless communication system can increase spectral efficiency. Furthermore, to achieve high data rate wireless communications, space-time communication systems need to be in wideband frequency selective channels. Orthogonal frequency division multiple (OFDM) has become a popular technique for transmission of signals over wireless channels. OFDM are robust to frequency selective fading channels for OFDM systems use the discrete Fourier transform (DFT) to modulate the data on orthogonal frequency carriers and effectively divide the wideband channel into a number of narrowband flat channels. One important advantage of the OFDM transmission technique is that the intersymbol interference (ISI) can be removed if the channel delay spread is less than he inserted guard interval.

Clearly, conventional MIMO detection algorithms can be applied for MIMO-OFDM system [1-4]. The MIMO maximum likelihood detection detector is the optimal receiver, but its complexity is best high. A number of sub-optimum receivers of low to moderate complexity have been devised, yet all suffer from rather limited performance. The conventional VBLAST algorithm exhibits the best tradeoff between performance and complexity.
However, it involves an intensive computation and hence it may be difficult to implement it for high data rate communications. Linear ZF and MMSE have the best low complexity but the performances are the worse. The QR [3] detection receiver avoids the matrix inversion, but the performance is not good as ZF-VBLAST. The QR detection based on ordering MMSE criterion is proposed [5]. Compared to zero-forcing criterion the detection method based on MMSE criterion needs estimating the signalnoise ratio or variance of noise.

Now considerable research interests have been focused on techniques and algorithms which realize various benefits of turbo principle for MIMO systems [6-8]. The method based on turbo principle, is regarded as an essential technique to furthermore improve system performance with soft iterative detection and decoding through an exchange of information. One of major drawbacks of such turbo-MIMO concepts is that its complexity increases exponentially with the number of transmit/receive antennas, the number of bits per symbol and /or the code constraint length.

However, the research work about iterative detection and decoding based on hard information is very litter. The hard iterative detection method has markedly advantage to soft iterative method in complexity. In this paper, we consider MIMO-OFDM systems with hard iterative detection process. In [9], joint processing of zero-forcing 
detection and MAP decoding for MIMO-OFDM system. Inspired by $[9,10]$, we introduce a new iterative detection receiver. This approach first utilizes parallel interference cancellation to detect signals of all layers, while the detected signals are regarded as input of channel decoder. For improving the performance of allover system, the output of decoder is regarded as input of PIC detection to do PIC again. By exchanging information between the MIMO detection and decoder, the performance of receiver may greatly be enhanced. Computer simulation result states the performance of proposed detection scheme is better than conventional coded MIMO-OFDM system.

The rest of this paper is organized as follows. In Section 2, we describe MIMO-OFDM systems model. In Section 3, a joint iterative detection and decoding scheme is proposed for MIMO-OFDM systems based on parallel interference cancel (PIC). The simulation results and performance analysis are presented in Section 4 and 5. Concludes follow in Section 6.

\section{MIMO-OFDM Systems Model}

Before introducing the signal detection, we briefly describe a MIMO-OFDM system. The combination of OFDM and VBLAST can overcome intersymbol inter- ference in frequency selective fading channels. A multicarrier system can be efficiently implemented in discrete time using an inverse FFT (IFFT) to act as a modulator and an FFT to act as a demodulator. The VBLAST architecture is based on a single carrier signal processing algorithm. Therefore, to combine it with OFDM, the VBLAST detection process has to be performed on every subcarrier at the receiver. The detailed system configuration of the VBLAST-OFDM is shown in Figure 1-2.

\subsection{MIMO-OFDM Systems}

In this section, we consider a coded MIMO-OFDM communication system with $n_{T}$ transmit antennas and $n_{R}$ receive antennas, denoted by $\left(n_{T}, n_{R}\right)$. Figure 1 is diagram of MIMO-OFDM transmitter. At the transmitter the input bit stream is de-multiplexed and coded to generate $n_{T}$ symbol streams. The encoded data stream is then interleaved and launched into the IFFT modulators and added cyclic prefix (CP). Finally, the OFDM signals are transmitted over every transmit antenna.

Figure 2 shows the block diagram of a VBLASTOFDM receiver. Each receiver antenna receivers signals sent from all transmit antennas. After the cyclic prefix is removed, each received signal passes through a FFT block for demodulation.

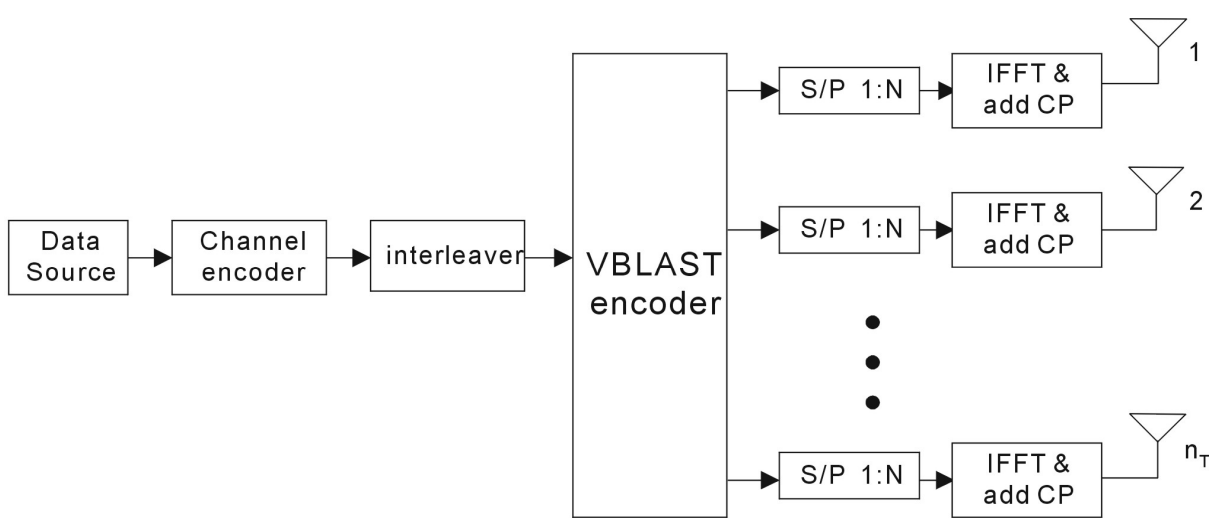

Figure 1. VBLAST-OFDM transmitter.

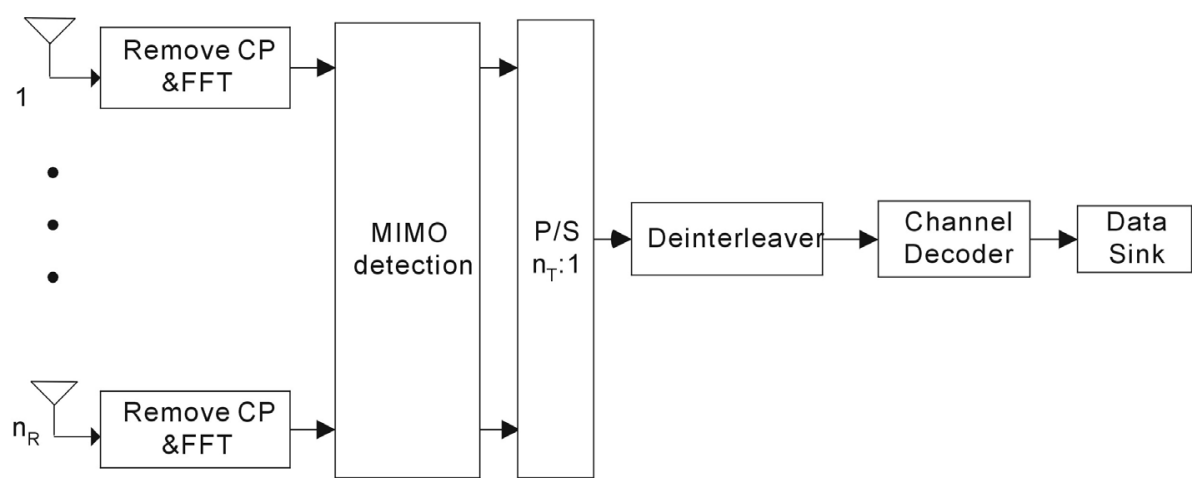

Figrue 2. VBLAST-OFDM receiver. 


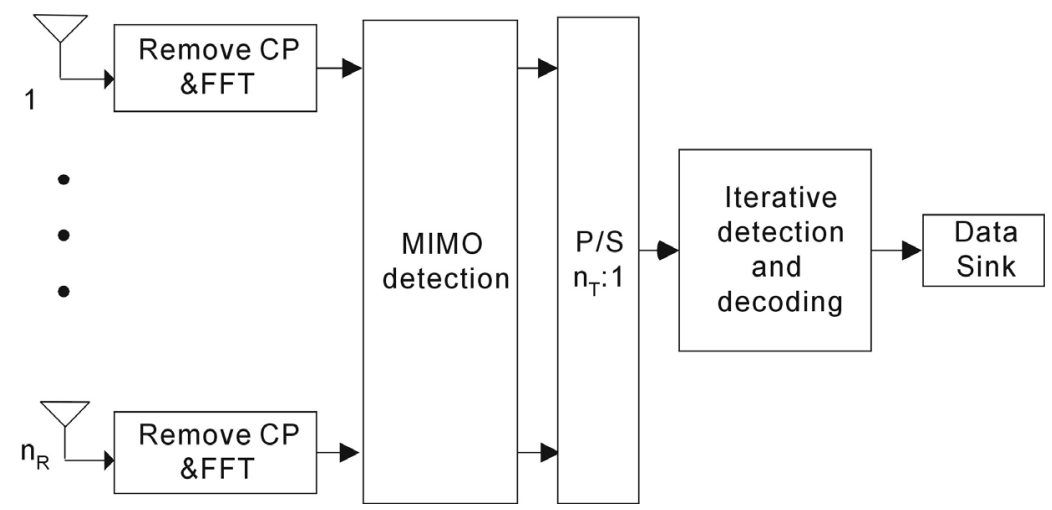

Figure 3. Proposed receiver structure for MIMO-OFDM system.

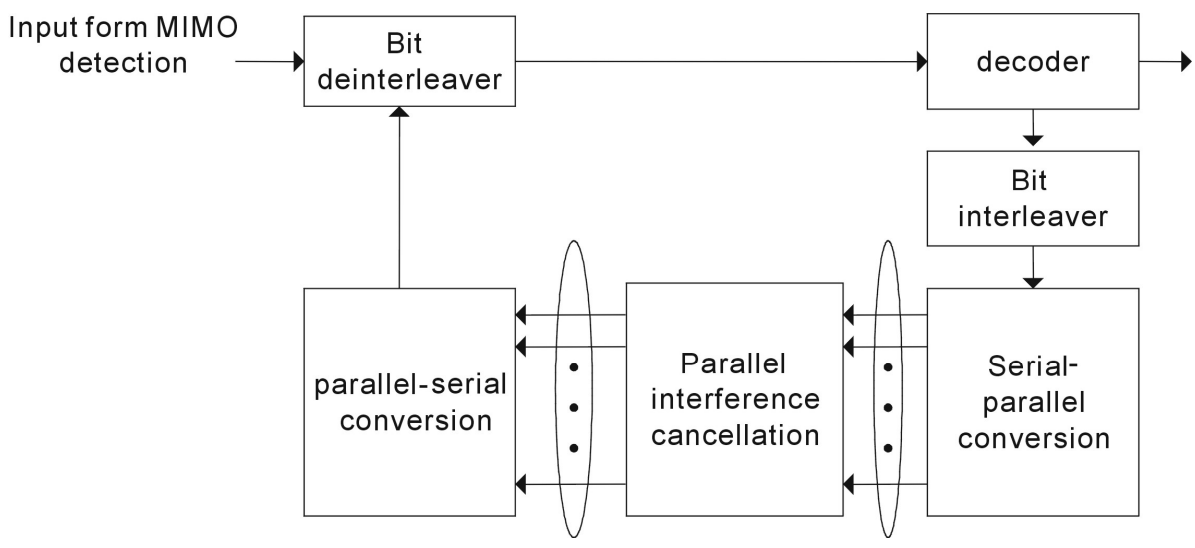

Figure 4. Proposed detection block.

At the receiver, we assume perfect OFDM synchronization. The receiver signal after demodulation, at receive antenna $j$ for subchannel $k$, is given by

$$
r_{j}[n, k]=\sum_{i} H_{i j}[n, k] s_{i}[n, k]+w_{j}[n, k]
$$

where $i=1, \cdots, n_{T}, j=1, \cdots, n_{R}, s_{i}[n, k]$ is the transmitted symbol from the $\mathrm{i}$-th transmit antenna at $\mathrm{k}$ subchannel, $w_{j}[n, k]$ in (1) denotes the additive complex Gaussian noise at the $j$ th receiver antenna, and is assumed to be zero-mean with variance $\sigma_{n}^{2} \cdot H_{i j}[n, k]$ in (1) denotes the channel frequency response for the $k$ th tone at time $n$, corresponding to the $i$ th transmit and the $j$ th receiver antenna.

During the reception, each receiver antenna receives the signal transmitted from all the $n_{T}$ transmit antennas. First, the cyclic prefix of each received signal is removed. After passing through a serial-to-parallel converter and the fast Fourier transformation blocks (FFTs), the subcarriers are separated. Then, the $\mathrm{N}$ information symbols belonging to each subcarrier are routed to their corresponding VBLAST multi-antenna processing unit where the de-mapping and decoding are performed. The detected bits are converted back into a serial form accordingly to recover the transmitted data bits.

\subsection{Channel Models}

We assume that the OFDM signal is transmitted over a wireless communications environment by a mutipah fading channel and a given coherence bandwidth. The complex baseband equivalent of a fading channel impulse response from transmit antenna $i$ to receiver antenna $j$ can be expressed as,

$$
h_{i j}(t, \tau)=\sum_{l=1}^{L} \alpha_{i j}^{l}(t) \delta\left(\tau-\tau_{l}\right)
$$

where $\alpha_{i j}^{l}(t)^{\prime} s$ are wide-sense stationary narrowband complex Gaussian processes and are assumed to be independent among different paths form transmit antenna $i$ to receiver antenna $j . \mathrm{L}$ is the number of multipaths. Thus for each receive antenna, the channel frequency response for the $k^{\text {th }}$ subcarrier at time $\mathrm{n}$ is

$$
H_{i j}[n, k]=\sum_{l=0}^{L-1} \alpha_{i j}^{l}(t) e^{-j 2 \pi k \Delta f \tau_{l}}
$$


where $\Delta f=\frac{B}{K}$ is the subcarrier spacing, $B$ is the total bandwitdth, and $K$ is the total number of subcarriers.

\section{Proposed Scheme}

To the above the VBLAST-OFDM system model, we proposed the new detection scheme for MIMO channel matrix at every sub-carrier. To reduce the complexity and prevent error propagation, the MIMO PIC detection is applied to obtain the initial estimation of transmitting signals for MIMO-OFDM architecture. Furthermore, iterative detection and decoding is used to enhance the performance of system in a progressive fashion.

Figure 3 presents a simple schematic of the proposed receiver scheme with PIC MIMO detection for MIMO-OFDM systems. For a wideband system, the OFDM demodulator is applied for each transmitter stream over $N$ parallel subchannels. To perform the iterative detection and decoding processing, the estimated signal by decoder is used to reconstruct the transmitted coded signal. Consequently, the PIC detection uses the reconstructed signal to improve the detection performance and start the iterative process. Figure .4 illustrates the proposed iterative detection and decoding block in Figure 3 in detail.

The PIC in [11] is another conventional detection algorithm which detects all layers simultaneously by subtracting interference from all the other layers regenerated by the estimate from hard decision based on ZF or MMSE criteria. The PIC detection scheme based on MIMO system algorithm is described as below:

Input $\boldsymbol{H}, \boldsymbol{r}$

$$
\begin{aligned}
& \tilde{\boldsymbol{s}}=\boldsymbol{H}^{+} \boldsymbol{r} \\
& \tilde{\boldsymbol{s}}=\mathrm{Q}(\boldsymbol{G} \boldsymbol{r}) \\
& \text { For } \mathrm{i}=1: n_{T} \\
& r=r-\sum_{\substack{j=1 \\
j \neq i}}^{n_{T}-1} H(:, j) \cdot \tilde{s}_{j} \\
& \boldsymbol{G}=(\boldsymbol{H}(:, j))^{+} \\
& \tilde{\boldsymbol{s}}_{j}=Q(\boldsymbol{G} \cdot r)
\end{aligned}
$$

End

Output $\tilde{\boldsymbol{s}}$

where $(\bullet)^{+}$is operation of calculating inverse of matrix (or vector). Comparing to conventional VBLAST, the PIC algorithm doesn't need calculate the pseudo-inverse matrix of the channel, so the complexity of proposed receiver is reduced. However, the detection scheme doesn't obtain the gain with applying the odering of the layers. Below sections, the performance of proposed scheme is study.

\section{Simulations Results}

In this section, we investigate the characteristics of our proposed receiver through computer simulations. We assume a perfect channel estimation and synchronization at the receiver.

In simulations, BPSK is used as the subcarrier modulation. We evaluate the performance of MIMO-OFDM systems. The FFT size is 64 , and the cyclic prefix length is $\mathrm{L}=16$. For the $20 \mathrm{MHz}$ channel, channel assumes an exponentially decaying power delay profile with 4 multipaths which are sample-spaced, and assume the channel is constant during one packet. In the simulation, the antenna configuration consists of 4 transmit antennas, and 4 receive antennas and BPSK is used. For comparison, we also include the performance of V-BLAST and the performance of conventional PIC receiver in signal detection.

We first evaluate the performance of PIC algorithm in MIMO-OFDM without coding case. Figure 5 shows the comparison performance of the three detection methods. Simulation states that PIC detection scheme obtains about $6 \mathrm{~dB}$ gain than linear ZF method at error bit rate $10^{-3}$. At low SNR case, the PIC detection scheme outperforms the conventional V-BLAST detection. At high SNR, the advantage of the proposed scheme died down because the affect of error diffuse is taken off. The performance of conventional ZF-VBLAST detection is the best when the SNR is higher than $8 \mathrm{~dB}$. The linear ZF has the worst performance.

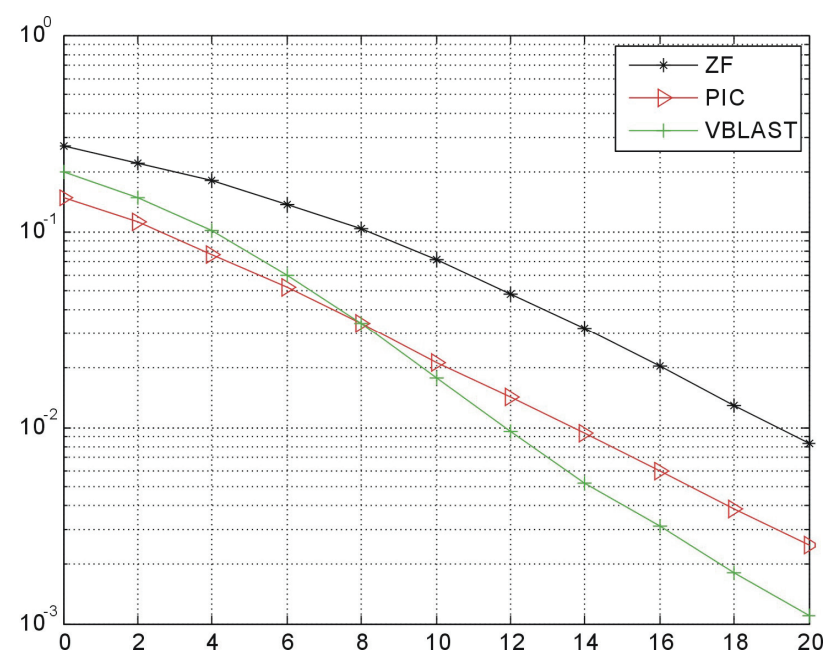

Figure 5. BER comparison for the $(4,4)$ MIMO-OFDM system with difference detection methods. 


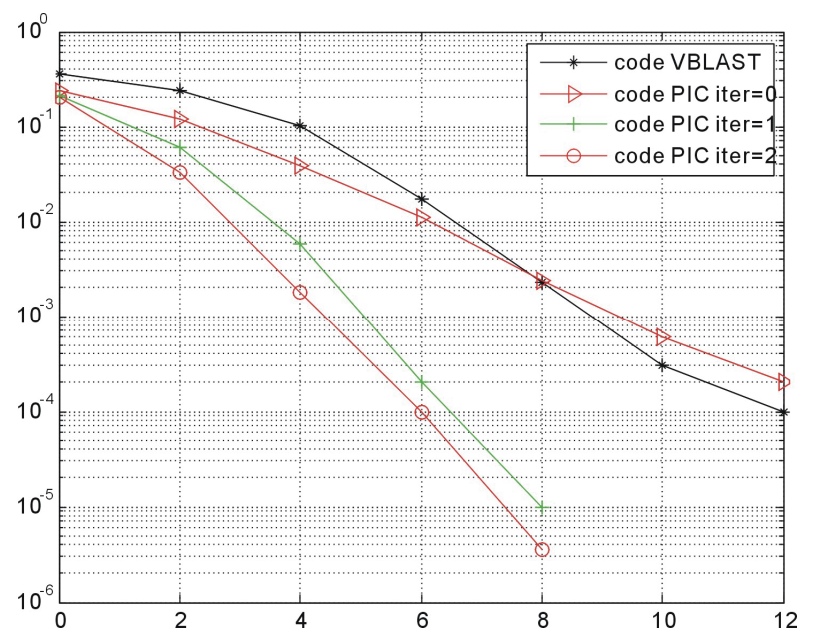

Figure 6. BER comparison for the $(4,4)$ code MIMO-OFDM system with difference detection methods.

Figure 6 shows the performance of proposed iterative detection and decoding scheme for code MIMO-OFDM systems. The convolutional code used in the simulations is a rate $1 / 2$, and the generator vectors are $g_{0}=(1,0,11)$ and $g_{1}=(1,1,0,1)$. The channel decoder uses the Viterbi algorithm. The other conditions is the same as to above case. As can be shown in this plot, the proposed MIMO detection scheme greatly outperforms the conventional linear Zero-Forcing detection.

The coded BER of proposed scheme is produced after $0,1,2$ iterations. When iteration is 0 , the proposed scheme is the conventional PIC algorithm for code MIMO-OFDM systems. We also observe that the proposed scheme outperforms the conventional coded PIC detection algorithm without iteration. After first iteration, the BER is significantly improved. There is a litter difference between the first iteration and the second iteration. Simulation states that proposed scheme with 2 iterations obtains about $4 \mathrm{~dB}$ gain than conventional MIMO-VBLAST algorithm without iteration at error bit rate 10-3.

From the simulation results presented in this section, the proposed scheme is quite effective in all simulation configurations. Below we furthermore analyze performance of proposed.

In actual, the difference algorithms have difference diversity order. For linear ZF receiver, the MIMO system may obtain $n_{R}-n_{T}-1$ order diversity gain and the conventional VBLAST algorithm may obtain $\geq n_{R}-n_{T}-1 \leq n_{R}$ order diversity gain [12]. However, PIC detection scheme may obtain $n_{R}$ order diversity gain when the interference comes from the all other layers is completely cancelled. Below we mainly analyze effect error propagation for our proposed detection scheme.

\section{Conclusions}

In this paper, we proposed a iterative detection and decoding scheme with PIC algorithm for MIMO-OFDM systems. To demonstrate the potential of this proposed technique, we have investigated the PIC and conventional VBLAST algorithms in MIMO-OFDM systems. We have also studied the effect of error forward coding on the system. By computer simulation, the performance of the proposed scheme is evaluated. The simulation results states that the performance of proposed scheme is greatly improved compared to conventional VBLAST detection receiver for MIMO-OFDM systems.

\section{References}

[1] P. W. Wolniansky, G. J. Roschini, G. D. Golden, and R. A. Valenzuela, "V-BLAST: An architecture for realizing very high data rates over the rich-scattering wireless channel," Proceedings International Symposium on Signals, Systems, and Electronics (ISSSE'98), Pisa, Italy, pp. 230-235, September 1998.

[2] D. Wubben, J. Rinas, R. Bohnke, V. Kuhn, and K. D. Kammeyer, "Efficient algorithm for detecting layered space-time codes," Proceedings ITG Conference on Source and Channel coding, Berlin, Germany, pp. 399-405, January 2002.

[3] B. Hassibi, "An efficient square-root algorithm for blast," in Proceedings IEEE International Conference Acoustic, Speech, Signal Processing, Istanbul, Turkey, pp. 5-9, June 2000.

[4] W. Zhu, J. Y. Jin, and Y. W. Park, "Detection algorithm improving parallel interference cancellation for V-BLAST system over error propagation," Proceedings ICACT2006, Gangwon-Do, Korea, pp. 11-15, February 2006.

[5] X. Li and X. Cao, "Low complexity signal detection algorithm for MIMO-OFDM systems," Electronics letters, Vol. 41, No. 2, pp. 20-21, January 2005.

[6] S. Haykin, M. Sellathurai, Y. D. Jong, and T. Willink, "Turbo-MIMO for wireless communications," IEEE Communications Magazine, Vol. 42, No. 10, pp. 48-53, October 2004

[7] M. Sellathurai and S. Haykin, "Turbo-BLAST for wireless communications: Theory and experiments," IEEE Transaction Signal Processing, Vol. 50, No. 10, pp. 2538-2546, October 2002.

[8] C. Berrou, A. Glavieum, and P. Thitimajshima, "Near shannon limit error-correcting coding and decoding: Turbo-Codes (1)," Proceedings of ICC'93, pp. 10641070, May 1993. 
[9] I. Sohn and J. Y. Ahn, "Joint processing of zero-forcing detection and MAP decoding for a MIMO-OFDM system,” ETRI Journal, Vol. 26, No. 5, October 2004.

[10] Z. P. Wang, "A group iterative QR receiver based on flat MIMO channels," in Proceeding IEEE WICOM2007, Shanghai, China, pp. 408-412, September 2007.
[11] W. H. Chin, A. G. Constantinides, and D. B. Ward, "Parallel multistage detection for multiple antenna wireless systems," IEEE Electronic letters, Vol. 38, No. 12, pp. 597-599, June 2002.

[12] A. Paulraj, R. Nabar, and D. Gore, "Introduction to space-time wireless communications," Cambridge University Press, pp. 153, 2003. 